\title{
Deep Cerebral Sinovenous Thrombosis Precipitated by High-Altitude Exposure
}

\author{
Muhib Khan, Angelos M. Katramados
}

Can. J. Neurol. Sci. 2010; 37: 700-702

Cerebral sinovenous thrombosis (CSVT) is rare and occurs in three to four persons per one million of population per year ${ }^{1}$. Deep cerebral venous system involvement is present only in $10 \%$ of CSVT $^{2}$. Thrombosis of deep cerebral veins presents with headache, encephalopathy, and variable focal deficits, and is associated with increased risk of death or dependence if not recognized and treated promptly ${ }^{3}$. Various risk factors have been identified for deep cerebral sinovenous thrombosis (DCSVT), of which oral contraceptive use, pregnancy and puerperium account for two thirds of these cases ${ }^{4}$.

Exposure to high altitude precipitating DCSVT is extremely rare with only one prior case report published in the English literature ${ }^{5}$. We report a case of a young male who developed DCSVT at high altitude.

\section{Case Report}

A 20-year-old previously healthy male with no significant past medical history was involved in an expedition in Ecuador at 4000 meters. Patient's hemoglobin before the expedition was 12 $\mathrm{g} / \mathrm{dl}$ and hematocrit was 0.32 before the expedition, respectively. Patient was also reported to be dehydrated while hiking on numerous occasions during the expedition. After two weeks of stay, the patient started having a severe holocephalic headache followed insidiously by confusion, and, eventually unresponsiveness.

At the time of presentation he was confused. He was mildly dehydrated as assessed by normal pulse, moist mouth and tongue and instant recoil of skin folds. Patient's hemoglobin and hematocrit was $18 \mathrm{~g} / \mathrm{dl}$ and 0.55 . Further examination revealed papilledema, right hemianopsia, right hemiplegia and right hyperreflexia with an extensor plantar response. Patient was intubated and transferred to a neurological intensive care unit for further management.

Non-enhanced CT of the head revealed hypodense areas in bilateral thalami, more notably on the left, with extension towards the left internal capsule and basal ganglia. There was evidence of edema bilaterally, but more prominently in the left thalamus, and associated hemorrhagic transformation. (Figure 1A). Magnetic resonance imaging of the brain revealed acute thrombosis of the deep venous system with extension into the torcula and the left transverse sinus (Figure 1B), bilateral thalamic hyperintensities on T-2 weighted images (Figure 1C), associated intraventricular and subarachnoid hemorrhage and early non-communicating hydrocephalus. MRV of the brain, and catheter angiography (Figure 1D) revealed thrombosis of bilateral internal cerebral veins, basal veins of Rosenthal, vein of Galen and straight sinus.

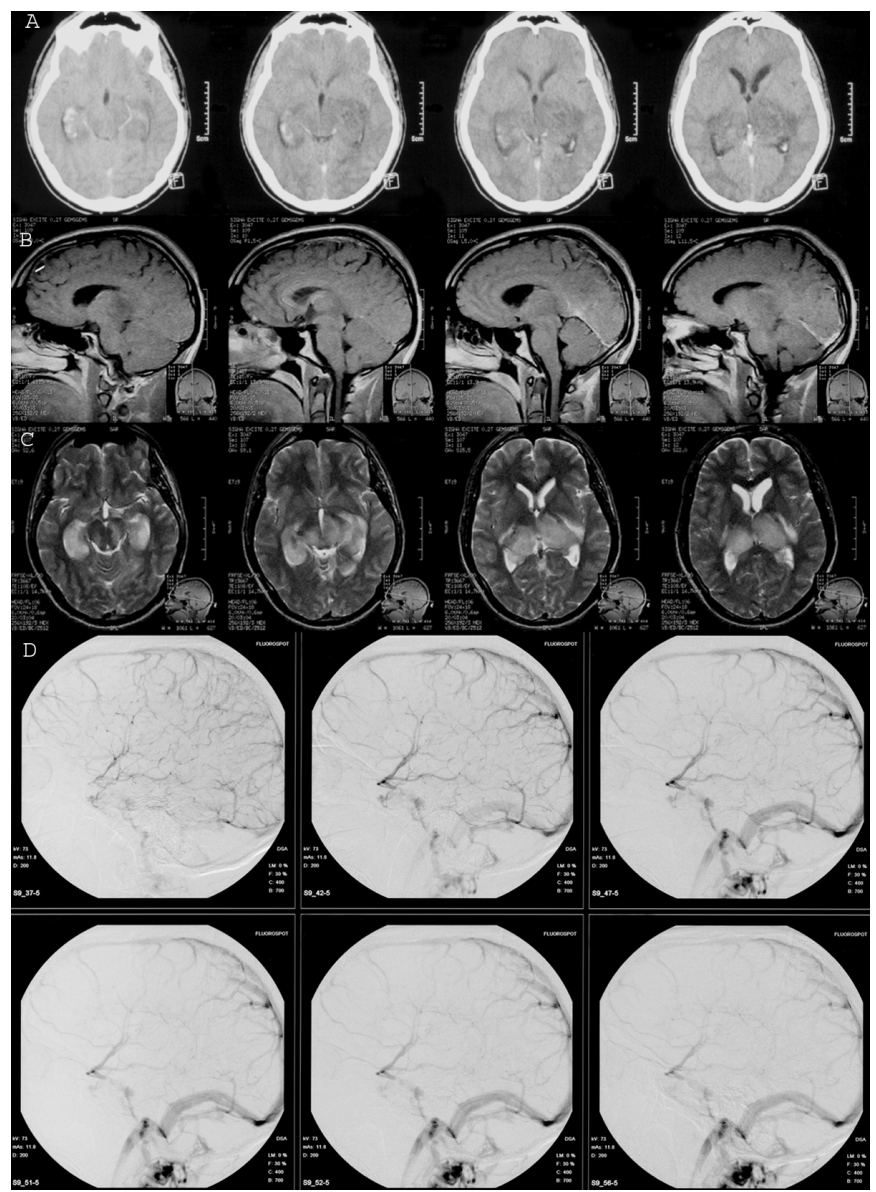

Figure 1: A) Non enhanced CT of the head demonstrating bilateral thalamic hypodensities, indicating edema and/or ischemia , B) MRI of the brain T1 sagittal images without gadolinium demonstrating acute thrombosis of the deep venous system extending to the torcula and the left transverse sinus and C) MRI of the brain T2 axial images without contrast demonstrating the parenchymal abnormalities, D) Catheter angiography with delayed venous phase imaging demonstrating the thrombotic process in the deep venous system.

From the Department of Neurology, Henry Ford Hospital, Detroit, Michigan, USA Received December 7, 2009. Final Revisions Submitted March 24, 2010. Correspondence to: Muhib Khan, Department of Neurology, Henry Ford Hospital, 2799 West Grand Blvd, Detroit, Michigan, 48202, USA 


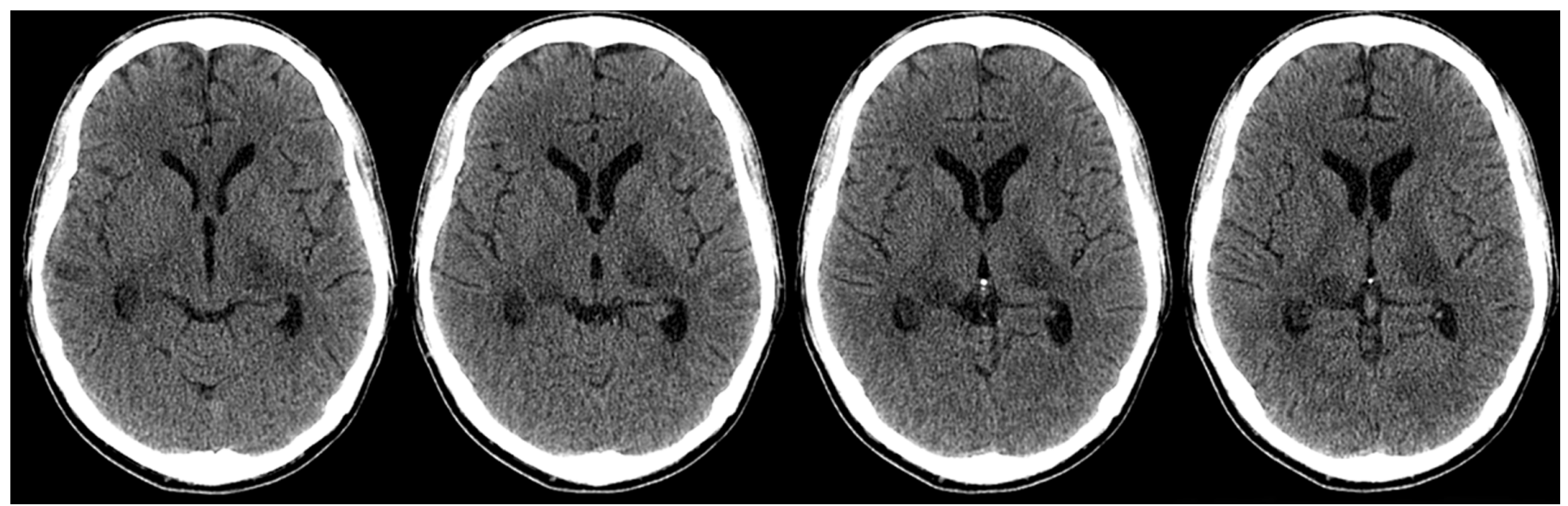

Figure 2: Follow-up non-Enhanced CT of the head demonstrating early interval decrease of the size of the thalamic hypodensities.

A diagnosis of DCVT was made and the patient was started on intravenous (IV) heparin. Moreover due to hydrocephalus, an external ventricular drain was inserted to relieve cerebral edema. Intravenous fluid resuscitation was initiated to improve mild dehydration and polycythemia. His hemoglobin and hematocrit progressively improved to $13 \mathrm{~g} / \mathrm{dl}$ and 0.44 respectively. Laboratory testing demonstrated factor $\mathrm{V}$ (Leiden) mutation in heterozygous form, and associated abnormal activated protein $\mathrm{C}$ resistance. Patient was negative for prothrombin 20210A mutation, MTHFR 677C->T variant.

The patient's presentation was attributed to high-altitude exposure and possible dehydration, resulting in secondary polycythemia, in the setting of a previously unknown genetic prothrombotic state. Patient was continued on chronic anticoagulation with oral warfarin. Repeat CT scan ten days later revealed improvement of the thalamic hypodensities and minimal residual hydrocephalus (Figure 2). Patient's hemiplegia also improved to a Medical Research Council strength of 4/5 in right leg and $3 / 5$ in the right arm. The external ventricular drain was removed and the patient was transferred to an inpatient rehabilitation facility. Modified Rankin Score at the time of discharge was 3. Follow-up MRI of the brain two months later showed significant interval improvement (Figure 3). Patient returned to clinic two months after discharge with residual deficits of right hemianopsia and increased spasticity in the right upper and lower extremities. Modified Rankin Score in the clinic was assessed to be 1 .

\section{Discussion}

The risk factors for DCSVT such as hypercoagulable state, oral contraceptives, pregnancy, malignancy, dehydration and infection are similar to those of $\mathrm{CSVT}^{6}$. Clinical manifestations are headache, focal deficits, altered consciousness and neuropsychological dysfunction ${ }^{7}$, as seen in our patient. These

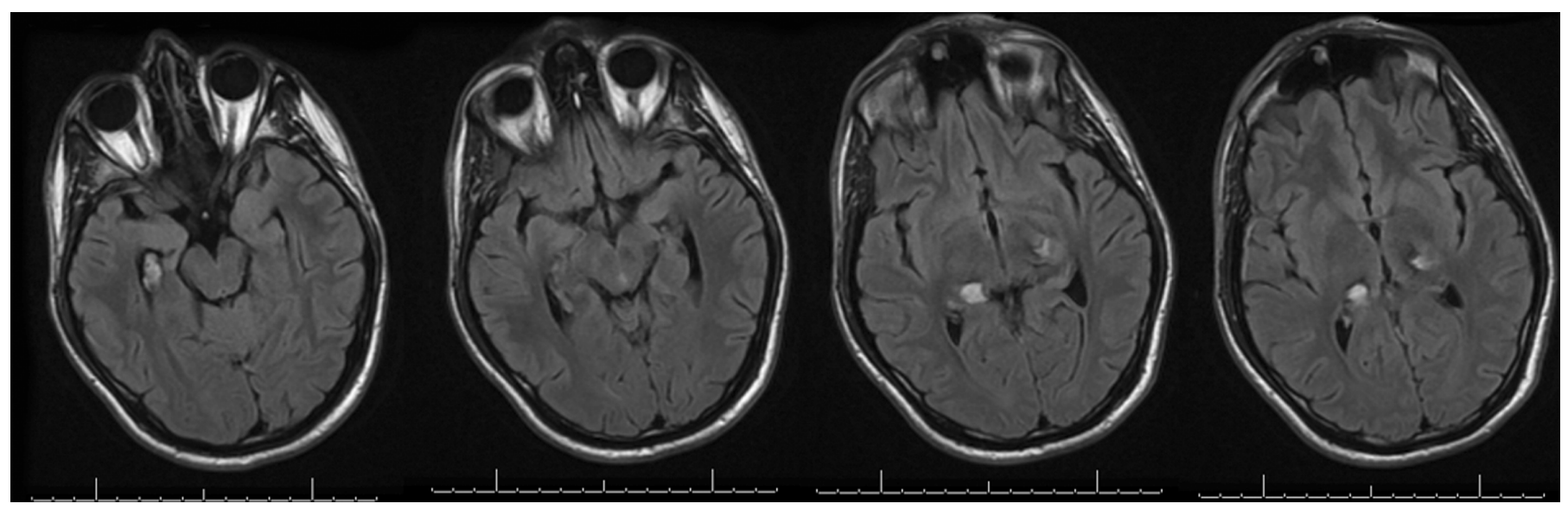

Figure 3: Follow-up MRI (T2 FLAIR) of the brain demonstrating chronic thalamic hemorrhages with resolution of most other parenchymal abnormalities. 
may develop acutely or insidiously, and are primarily due to the fact that DCSVT affects structures constituting the diencephalon, which regulates consciousness.

Non-enhanced CT is a useful tool but only shows abnormalities in $30 \%$ of cases. Hypodensities in the thalamic and basal ganglia regions, especially when bilateral, should raise suspicion for possible DCSVT. Magnetic resonance imaging of the brain may detect parenchymal abnormalities related to ischemia, edema, or hemorrhage. Conventional cerebral angiography with delayed venous phase imaging is the gold standard for depiction of the vessel thrombosis. MRV is a noninvasive modality which should be considered, but lacks sensitivity with regard to involvement of the deep venous system $^{8}$.

The clinical course of these patients is worsened by hydrocephalus and hemorrhagic transformation, as demonstrated in our patient. Careful anticoagulation with IV heparin is the mainstay of treatment, and should be initiated as early as possible, even in the presence of hemorrhagic transformation ${ }^{3,8}$. It has been shown to reduce death and dependence ${ }^{9}$. In patients not improving clinically on heparin, endovascular thrombolytic therapy might be beneficial, although clinical trials are still needed to validate its use ${ }^{10}$. Patients who develop headache due to intracranial hypertension secondary to chronic CVST can be treated with acetazolamide and with cerebrospinal fluid shunt procedures if needed ${ }^{11}$.

Few cases of CSVT due to high altitude have been reported earlier in the literature ${ }^{5,12-14}$, with only one prior involving the deep venous system. In contrast, studies have reported a high incidence (40\%) of deep venous system involvement in pediatric cerebral venous thrombosis. This is an important consideration keeping in view that our patient is only 20 -years-old ${ }^{15}$. The main reason was speculated to be polycythemia, but some patients also had concurrent protein $\mathrm{C}$ or $\mathrm{S}$ deficiency ${ }^{12}$. Kotwal et al showed that there was a significant rise from baseline in hemoglobin, platelet count and fibrinogen levels in soldiers spending eight months at 3500 meters producing a presumed hypercoagulable state $^{16}$. Based on current evidence, recommendations for routine testing of familial thrombophilia cannot be made, but mountaineers are advised to maintain adequate hydration, and avoid smoking.

As a conclusion, DCSVT/CSVT may be precipitated by highaltitude exposure especially in the setting of a genetic predisposition. The clinical presentation may be elusive and a high index of suspicion is required. Advances in MRI have allowed for earlier and more accurate diagnosis. Anticoagulation is the treatment of choice, while endovascular therapies can be considered, if the thrombotic process cannot be contained with anticoagulation alone.

\section{REFERENCES}

1. Stam J. Thrombosis of the cerebral veins and sinuses. N Engl J Med. 2005;352:1791-8.

2. van den Bergh WM, van der Schaaf I, van Gijn J. The spectrum of presentations of venous infarction caused by deep cerebral vein thrombosis. Neurology. 2005;65:192-6.

3. Ferro JM, Canhao P, Stam J, Bousser MG, Barinagarrementeria F. Prognosis of cerebral vein and dural sinus thrombosis: results of the International Study on Cerebral Vein and Dural Sinus Thrombosis (ISCVT). Stroke. 2004;35:664-70.

4. Brown JI, Coyne TJ, Hurlbert RJ, Fehlings MG, Ter Brugge KG. Deep cerebral venous system thrombosis: case report. Neurosurgery. 1993;33:911-3.

5. Cheng S, Chng SM, Singh R. Cerebral venous infarction during a high altitude expedition. Singapore Med J. 2009;50:e306-8.

6. Ehtisham A, Stern BJ. Cerebral venous thrombosis: a review. Neurologist. 2006;12:32-8.

7. Haley EC Jr, Brashear HR, Barth JT, Cail WS, Kassell NF. Deep cerebral venous thrombosis. Clinical, neuroradiological, and neuropsychological correlates. Arch Neurol. 1989;46:337-40.

8. Bousser M-G, Ross Russell RW. Cerebral venous thrombosis. London: WB Saunders; 1997.

9. Stam J, de Bruijn S, deVeber G. Anticoagulation for cerebral sinus thrombosis. Stroke. 2003;34:1054-5.

10. Ciccone A, Canhao P, Falcao F, Ferro JM, Sterzi R. Thrombolysis for cerebral vein and dural sinus thrombosis. Cochrane database of systematic reviews (Online) 2004:CD003693.

11. Bousser MG, Ferro JM. Cerebral venous thrombosis: an update. Lancet Neurol. 2007 Feb;6(2):162-70. Review.

12. Fujimaki T, Matsutani M, Asai A, Kohno T, Koike M. Cerebral venous thrombosis due to high-altitude polycythemia. Case report. J Neurosurg. 1986;64:148-50.

13. Jha SK, Anand AC, Sharma V, Kumar N, Adya CM. Stroke at high altitude: Indian experience. High Alt Med Biol. 2002;3:21-7.

14. Boulos P, Kouroukis C, Blake G. Superior sagittal sinus thrombosis occurring at high altitude associated with protein $\mathrm{C}$ deficiency. Acta Haematol. 1999;102:104-6.

15. deVeber G, Andrew M, Adams C, Bjornson B, Booth F, Buckley DJ, et al. Canadian Pediatric Ischemic Stroke Study Group. Cerebral sinovenous thrombosis in children. N Engl J Med. 2001 Aug 9;345(6):417-23.

16. Kotwal J, Apte CV, Kotwal A, Mukherjee B, Jayaram J. High altitude: a hypercoagulable state: results of a prospective cohort study. Thromb Res. 2007;120:391-7. 